\title{
CHINESE HERBAL MEDICINE SHENQI DETOXIFICATION GRANULE INHIBITS FIBROSIS IN ADENINE INDUCED CHRONIC RENAL FAILURE RATS
}

\author{
Min Peng ${ }^{1}$, Pingping Cai ${ }^{1}$, Hongbo Ma ${ }^{1}$, Hongyan $\mathrm{Meng}^{1}$, Yuan $\mathrm{Xu}^{1}$, Xiaoyi Zhang ${ }^{1}$ and Guomin $\mathrm{Si}^{1}{ }^{1}$. \\ Department of Traditional Chinese Medicine, Provincial Hospital Affiliated to Shandong University, 324, Jinwu Weiqi \\ Road, Ji'nan, 250021, China. \\ *E-mail: sgm977@126.com
}

\begin{abstract}
Background: Progressive fibrosis accompanies all chronic renal disease, connective tissue growth factor (CTGF,) and platelet-derived growth factor-B, (PDGF-B,) play important roles in extra-cellular matrix abnormal accumulation, while endothelin -1 (ET-1) nitric oxide (NO,) are related to endothelial dysfunction, which mediates the progression of renal fibrosis. Shenqi Detoxification Granule (SDG), a traditional Chinese herbal formula, has been used for treatment of chronic renal failure in clinic for many years.

Materials and Methods: In order to evaluate the efficacy, and explore the mechanism of SDG to inhibit the progression of renal fibrosis, study was carried out using the adenine-induced Wister rats as the CRF model, and losartan as postive control drug. Levels of serum creatinine [Scr], and blood urea nitrogen (BUN), albumin (ALB), 24hrs, urine protein (24hUP), triacylglycerol (TG), and cholesterol (CHO), together with ET-1, and NO were detected. Pathological changes of renal tissues were observed by HE, staining. In addition, CTGF and PDGF-B expression were analyzed by immuno-histo-chemistry.
\end{abstract}

Results:The results indicated that SDG can effectively reduce Scr, BUN, 24hUP, TG, and CHO levels, increase ALB levels, inhibit renal tissue damage in CRF rats, and the mechanism maybe reduce PDGF-B, CTGF expression and ET-1/NO.

Conclusion: Shenqi Detoxification Granule is a beneficial treatment for chronic renal failure.

Key words: Chronic Renal Failure; fibrosis; Shenqi Detoxification Granule; CTGF; PDGF-B.

\section{Introduction}

Many renal diseases in humans are progressive in nature, and the process irreversible; inevitably leading to end-stage renal failure, a condition that requires life long dialysis or renal transplantation. Thus, the number of patients with renal failure continues to be on the increase globally, and represents a relevant medical and financial burden. As costs of renal diseases are substantial for all health systems around the world, early detection of kidney diseases, followed by prevention of end-stage renal disease is essential. Therefore, there is need for the development of new effective therapeutic strategies.

Traditional Chinese medicine (TCM), is a well defined and established therapeutic system, and has widely been used in the treatment of various diseases in China for thousands of years. Shenqi Detoxification Granule (SDG), a traditional Chinese herbal formula, had been used for treatment of chronic renal failure in clinics for many years. With the function of tonifying the kidney, invigorating blood circulation and detoxifcation according to TCM, SDG could decrease both blood Urea nitrogen (BUN), and serum creatinine (Scr), postpone the aggravation of the renal function, and delay the progress of chronic renal failure (CRF), in our previous study (Li et al., 2007; Zhang et al., 2008; Li et al.,2010). However, mechanism of SDG in inhibiting the progression of renal fibrosis is yet to be addressed.

The progressive fibrosis accompanies all chronic renal disease. An overview of renal disease suggests that complementary but different mechanisms are responsible for fibrosis (Hewitson, 2012). Fibrosis involves an excess accumulation of extracellular matrix (ECM), and usually results in loss of function when normal tissue is replaced with scar tissue (Wynn, 2007). CTGF, (connective tissue growth factor) and PDGF-B( platelet derived growth factor-B) play important roles in ECM abnormal accumulation. CTGF has been shown to modulate many signaling pathways leading to cell adhesion and migration, angiogenesis, myofibroblast activation, and ECM deposition and remodeling, which together lead to tissue remodeling, and fibrosis (Kenneth et al.,2012). PDGF, in particular or the PDGF-B chain, is known to induce glomerular mesangial cell proliferation, and ECM protein accumulation in vitro (Doi et al., 1992; Floege et al., 1995; Throckmorton et al., 1995). Endothelin 
http://dx.doi.org/10.4314/ajtcam.v11i1.31

-1 (ET-1), and nitric oxide (NO), are antagonistic vaso-active substances, and their balance plays an important role in regulation of blood pressure, renal blood flow and glomerular filtration rate. Chronic hypoxia mediates the progression of renal fibrosis, even from the early stages of chronic kidney disease (CKD) (Mimura et al., 2010).

In order to further evaluate the efficacy of this Chinese herbal medicine, and explore the possible mechanism, we had done some experiments using the adenine-induced Wister rats as the CRF, model. Losartan, the Ang $\square$ receptor antagonist, is efficient in preventing renal fibrosis (Dussalue et al., 2000), and was chosen as positive control drug. The rats were treated with SDG, and losartan. Levels of serum creatinine (Scr), and blood urea nitrogen (BUN), albumin (ALB), 24hrs, urine protein (24hUP), triacylglycerol (TG), and cholesterol (CHO), together with ET-1, and NO, were detected. Pathological changes of renal tissues were observed by HE staining. In addition, CTGF, and PDGF-B expression were analyzed by immunohistochemistry.

\section{Materials and methods}

Experimental animals

The study was approved by the Institutional Animal Care and Use Committee of the Shandong Provincial Hospital. Experiment was performed using 80-8 to 10-week old, male Wistar rats (180 to 200g; Laboratory Animal Center, The Shandong University, China). Animals were maintained at a constant humidity (ca. $60 \%$ ), and temperature (ca. $23^{\circ} \mathrm{C}$ ), with a light/dark cycle of $12 \mathrm{hrs,} \mathrm{in} \mathrm{our} \mathrm{institutional} \mathrm{stable} \mathrm{with} \mathrm{free}$ access to food and water. After 7, days of acclimatization, animals were randomized into 4, groups: normal control group (NG), of 20, animals, CRF model group (MG), of 20, animals, losartan group (LG), of 20, animals, and Shenqi Detoxification Granual group (SG), of 20, animals. The rats in $\mathrm{NG}$ were fed a standard laboratory chow $(0.35 \mathrm{~g} \mathrm{NaCl}, 20 \mathrm{~g}$ protein) and allowed tap water ad libitum. We monitored body weight, food consumption, and fluid intake weekly.

\section{Induction of renal failure}

Renal failure was induced in rats except NG. 60, rats were fed with diet containing $0.75 \%$ adenine (Amresco, USA, Lot number: 0272B31), (Yokozawa et al., 1986; Johns et al., 1990). Except for the added adenine, all diets were of identical composition. After 4, weeks adenine feeding, all rats were fasted for $12 \mathrm{hrs}$, and then collected blood samples $1.5 \mathrm{ml}$ by cutting rats' tails to detect BUN and Scr. Data was analyzed statistically to confirm successful modeling. 1 rat in LG, SG died respectively in this process.

\section{Drug therapy}

After successful modeling, the rats in NG, and MG were given normal saline $2 \mathrm{ml}$ daily by gavage. Rats in LG were given losartan (50mg/pill, Lot number: H20000371, Hangzhou MSD Pharmaceutical Co., Ltd., and made into 0.5mg/ml liquid, kept at $4 \square$.), $5.1 \mathrm{mg} /(\mathrm{kg} \cdot \mathrm{d})$, by gavage once a day. Each rat in SG, was administered 4.1g/kg/d Shenqi Detoxification Granule solution by gavage once a day (Xu, 2002; Huang et al., 2004). Shenqi Detoxification Granual administered was made up as follows: Huang Qi[Radix Astragali]60g, Dan Shen[Salvia miltiorrhiza]20g, Dang Gui[Angelica sinensis]12g, Da Huang[Rheum officinale]10g, Qian Shi[gordon euryale seed]15g, Han Lian Cao[Herba Ecliptae]15g, Tian Kui Zi[radix semiaquilegiae]15g, Bai Mao Gen[rhizoma imperatae]15g, Shu Fu Zi[Prepared aconite lateral root]10g, Yin Yang Huo[Herb of Shorthorned Epimedium]15g, Jin Ying Zi[Cherokee Rose Fruit]15g, Bai Hua She She Cao[Hedyotis diffusa]15g. These 12 crude drugs were mixed in water, the quantity of water used for extract was $12,10,8$, times of the quantity of the herb respectively and the extract time was $1.5 \mathrm{~h} 、 2 \mathrm{~h} 、 2 \mathrm{hrs}$, respectively, then combined decoction, filtrated, and concentrated to a relative density of $1.30-1.35\left(50{ }^{\circ} \mathrm{C}\right),(1 \mathrm{ml}$ clear paste is equivalent to $2.5 \mathrm{~g}$ crude drugs). Clear paste and excipient were mixed to make sugar-free granule, then dried, granulated, packing (10g/bag), and labeling, which $20 \mathrm{~g}$, granule is quite to $217 \mathrm{~g}$, crude drugs. Then the granule was made into $0.4 \mathrm{mg} / \mathrm{ml}$ liquid, kept at $4^{\circ} \mathrm{C}$. The period of drug therapy lasted 8 weeks. 2 rats in MG and 1 rat in LG died in this process.

\section{Specimen collection}

2 days before the end of the experiment, the rats were placed in metabolic cages to collect 24-hour urine of rats, and recorded 24-hour urine volume to determine the 24 -hour urine protein amount ( 24 hours urine protein, $24 \mathrm{hUP}$ ). Fasting $12 \mathrm{hrs}$, after the last administration, all rats were 
http://dx.doi.org/10.4314/ajtcam.v11i1.31

anesthetized by $3 \%$, Sodium pentobarbital through intra-peritoneal injection, and $7 \mathrm{ml}$ blood samples were collected from heart under direct vision, wherein $5 \mathrm{ml}$ were injected into the test tube, and centrifuged at $1500 \mathrm{r} / \mathrm{min}$ for 10 minutes at room temperature, dispensed serum, set at -20 cryopreservation analyte. Further $2 \mathrm{ml}$ were injected into tubes containing $10 \%$ EDTA $\cdot$ Na230 $\mu \mathrm{L}$, and aprotinin $40 \mu \mathrm{L}$, $4 \square$, centrifuged at $3000 \mathrm{r} / \mathrm{min}$ for 10 minutes, frozen at $-20 \square$ refrigerator under test. After the final blood sampling, left ventricular were perfumed with saline $200 \mathrm{ml}$, until liver was gray, then neutral formaldehyde fixative $200 \mathrm{ml}$, used for systemic perfusion until the liver was hard. Both kidneys of each rat were isolated, and the renal weights were measured using a Sartorius Genius scale (Sartorius AG). Left kidneys were fixed with neutral formalin, paraffin-embedded and sliced. Right kidneys were shock-frozen with liquid nitrogen and stored at $-80^{\circ} \mathrm{C}$. The serum levels of BUN, Scr, TG, $\mathrm{CHO}$ and ALB were determined by Automatic Biochemical Analyzer (OLYMPUS AUS5400). Pathological changes of renal tissues were observed by HE staining. CTGF and PDGF-B were examined by immunohistochemical SP method, using Image-Plus image analysis system to analyze the results. NO was determined by nitrate reductase method, while the plasma ET-1 by radioimmunoassay.

\section{Data analysis}

The data were analyzed using the software SPSS 12.0 for Windows. Quantitative data and presented as mean \pm SD, and analyzed using student's t-test between two groups. $\mathrm{P}<0.05$ was considered significant.

\section{Results}

\section{Animal characteristics}

At the beginning of this study, all rats behaved normally. After 5-7, days, all rats fed with adenine gradually showed polydipsia, and polyuria, decreased food intake, lost weight, listlessness, tended to crouch, disheveled hair, and growth inhibition, even appeared; symptoms such as muscle tremors and convulsions, subsequently, 2 rats died in this process. After 8, weeks of drug therapy, except the normal group, the remaining rats still existed above phenomena at different degrees, and rats in MG gradually appeared as dry coat, pale ear, swollen eyelids, and clammy tail reduced activity. At the end of experiment, 2, rats in MG, and 1 rat in LG died in this process. The causes of death were related to complications or failure induced by uremia toxins; also the individual rats died due to lung infection caused by drugs introduced into the lungs in gavage.

\section{BUN and Scr decreased in SG}

After 4, weeks adenine feeding, BUN and Scr in model group were higher than those in NG $(\mathrm{P}<0.05)$, demonstrated severe susceptibility to adenine administration, which meant the successful modeling (Figure 1).

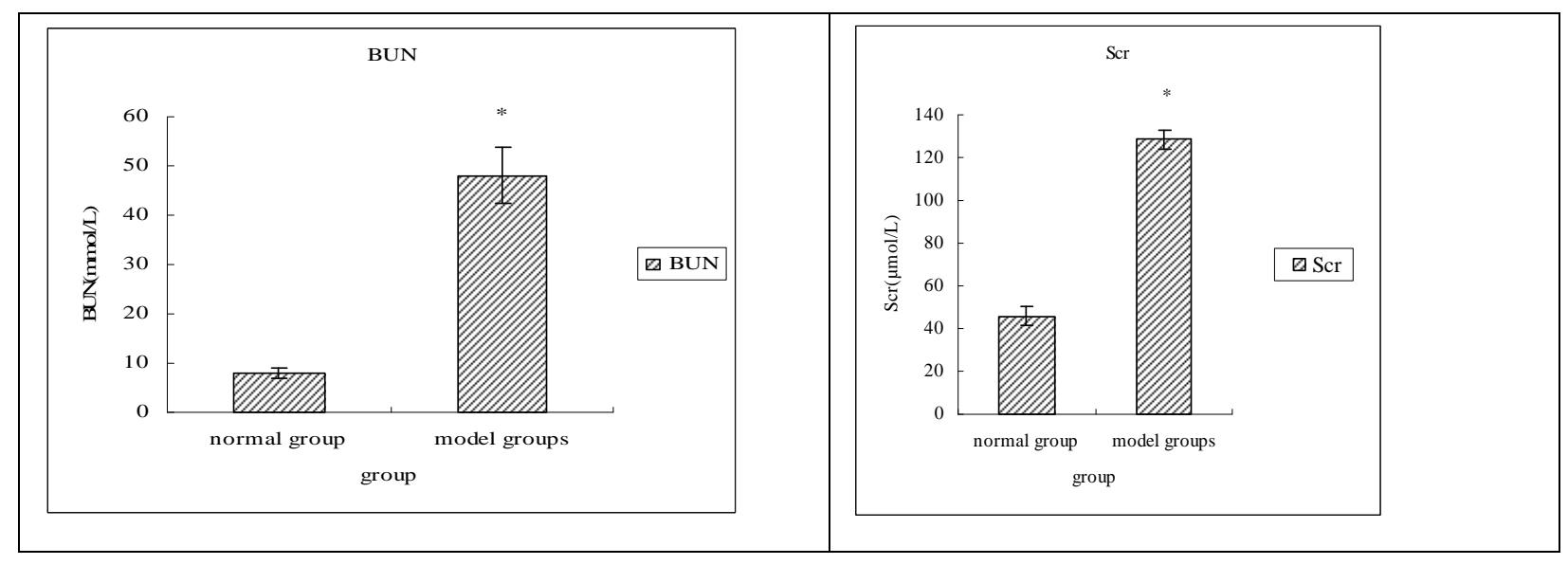

Data are expressed as mean \pm S.D. ${ }^{*} \mathrm{P}<0.05$, vs normal group.

Figure 1: BUN, and Scr levels of rats in normal group, and model groups after 4-wk adenine feeding. 
http://dx.doi.org/10.4314/ajtcam.v11i1.31

After 8, weeks drug therapy, BUN, and Scr in LG and SG were lower than those in MG $(\mathrm{P}<0.05)$, and all showed significant differences when compared with the levels before therapy $(\mathrm{P}<0.05)$. The levels in LG and SG had no difference (Figures 2,3 ).

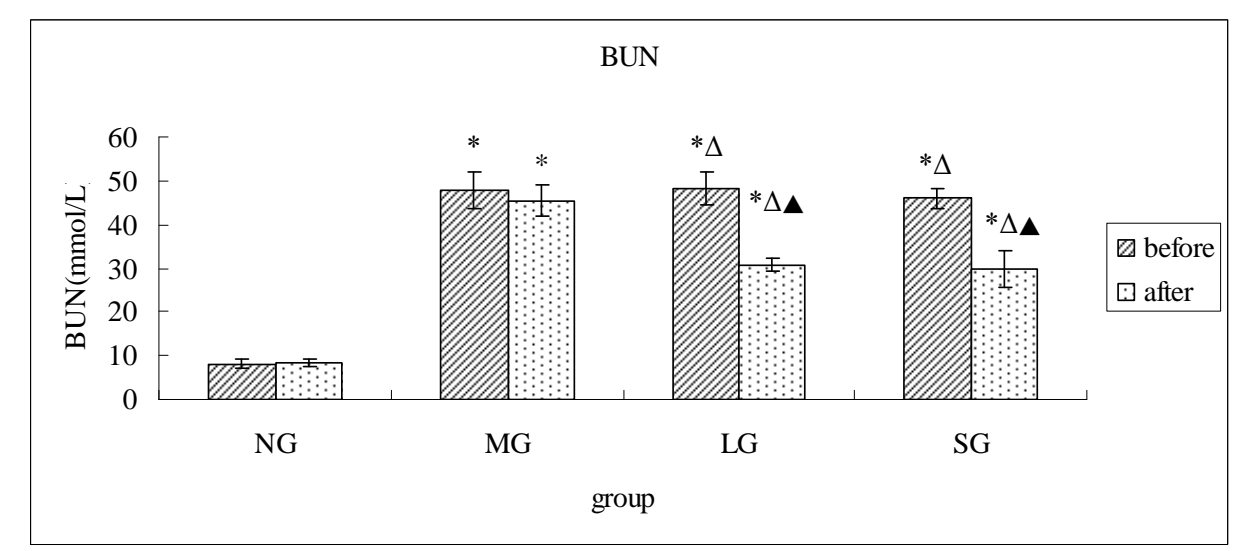

Data are expressed as mean \pm S.D. ${ }^{*} \mathrm{P}<0.05$, vs NG; ${ }^{\Delta} \mathrm{P}<0.05$, vs MG ; ${ }^{\Delta} \mathrm{P}<0.05$, vs before.

Figure 2: BUN levels of rats in different groups before and after drug therapy.

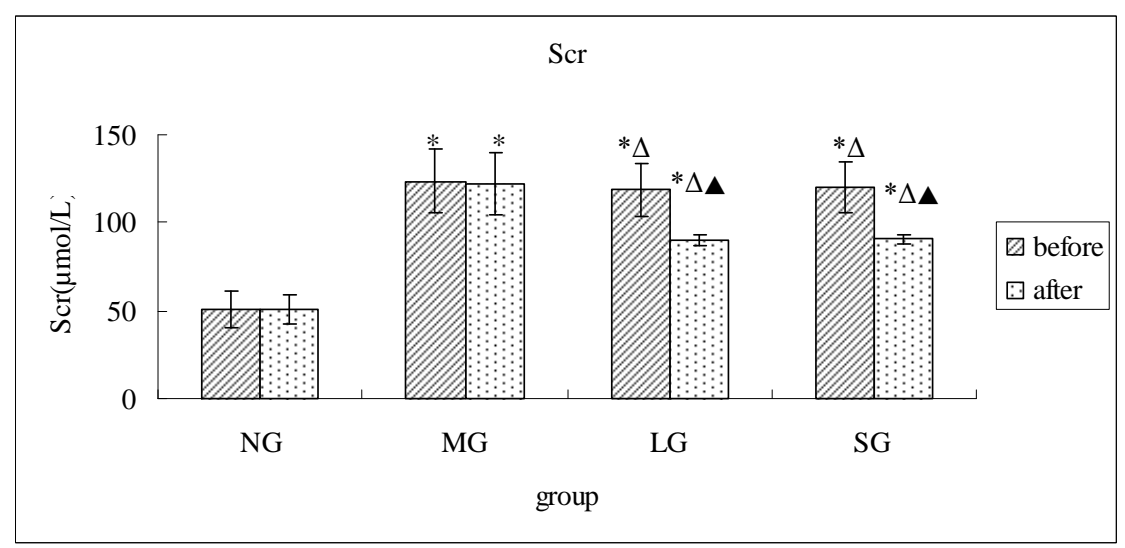

Data are expressed as mean \pm S.D. ${ }^{*} \mathrm{P}<0.05$, vs NG; ${ }^{\Delta} \mathrm{P}<0.05$, vs $\mathrm{MG} ;{ }^{\mathbf{\Delta}} \mathrm{P}<0.05$, vs before.

Figure 3: Scr levels of rats in different groups before and after drug therapy.

\section{Relevant biochemical markers improved in SG}

After 8, weeks therapy, ALB in adenine fed rats were lower, while 24UP were higher than those in NG ( $P<0.05)$. ALB in LG and SG were higher, and 24hUP were significantly lower than those in MG $(\mathrm{P}<0.05)$. The levels in LG and SG had no difference $($ Fig. 4,5$)$. 


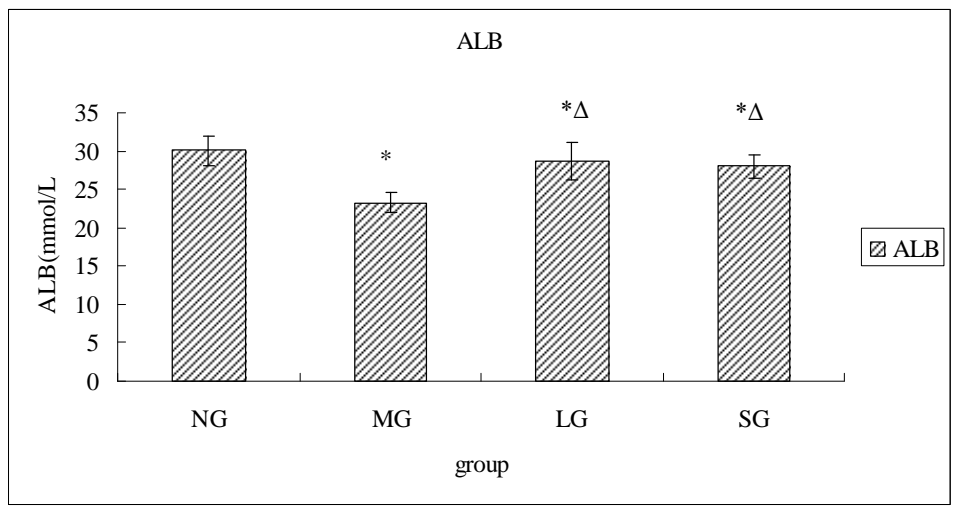

Data are expressed as mean \pm S.D. ${ }^{*} \mathrm{P}<0.05$, vs NG; ${ }^{\Delta} \mathrm{P}<0.05$, vs MG.

Figure 4: ALB levels of rats in different groups after therapy.

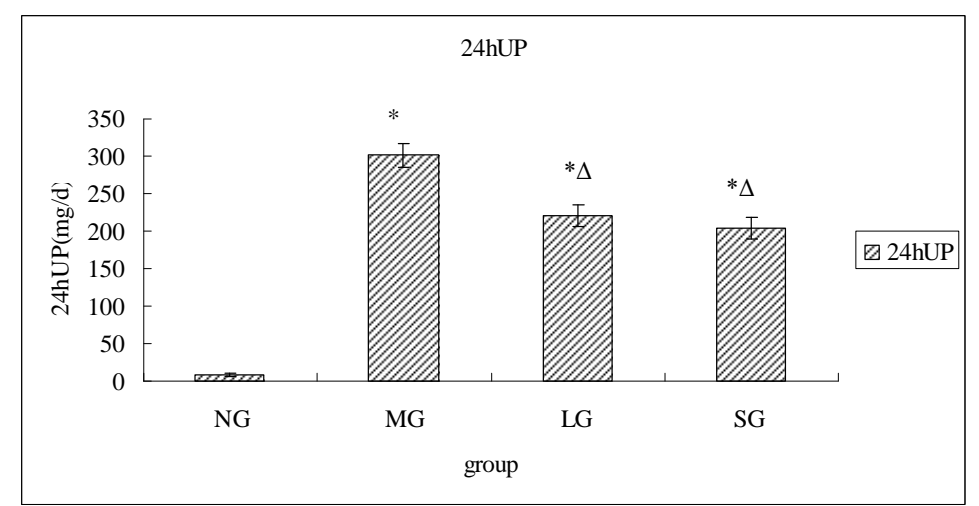

Data are expressed as mean \pm S.D. ${ }^{*} \mathrm{P}<0.05$, vs NG; ${ }^{\Delta} \mathrm{P}<0.05$, vs MG.

Figure 5: 24UP levels of rats in different groups after therapy.

TG and CHO in adenine fed rats were higher than those in NG, and the levels in LG and SG were higher than those in MG $(\mathrm{P}<0.05)$. The levels in LG and SG had no difference (Figure 6).

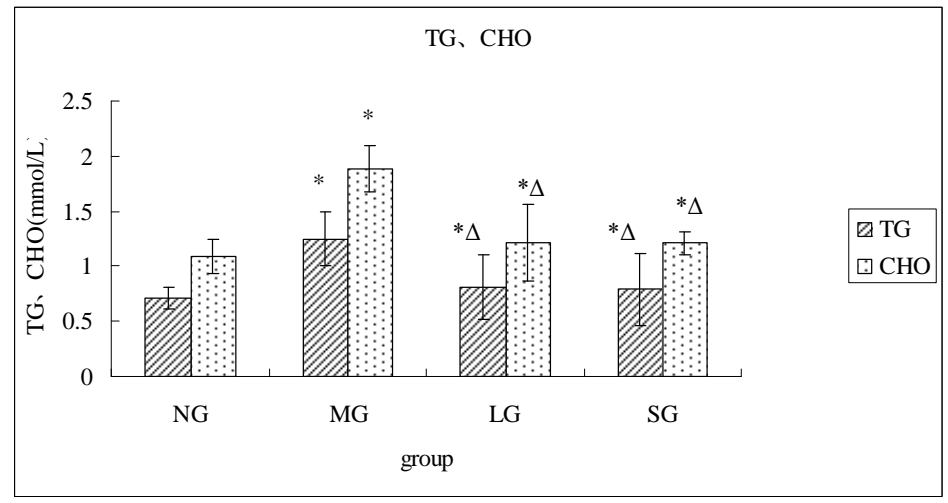

Data are expressed as mean \pm S.D. ${ }^{*} \mathrm{P}<0.05$, vs NG; ${ }^{\Delta} \mathrm{P}<0.05$, vs MG.

Figure 6: $\quad$ TG and $\mathrm{CHO}$ levels of rats in different groups after therapy.

\section{SG can inhibit further damage by adenine}

Renal pathology in adenine fed rats developed structural damages, included tubular dilatation, and epithelial hyperplasia; crystals of adenine in the interstitium, inflammatory cellular infiltration, and giant cells in interstitium. The changes in SG showed reduced 2,8-dihydroxy adenine crystal deposition in the renal tubules, and damaged cells were significantly recovered, which were superior to losartan (Figure 7). 
Peng et al., Afr J Tradit Complement Altern Med. (2014) 11(1):194-204

http://dx.doi.org/10.4314/ajtcam.v11i1.31
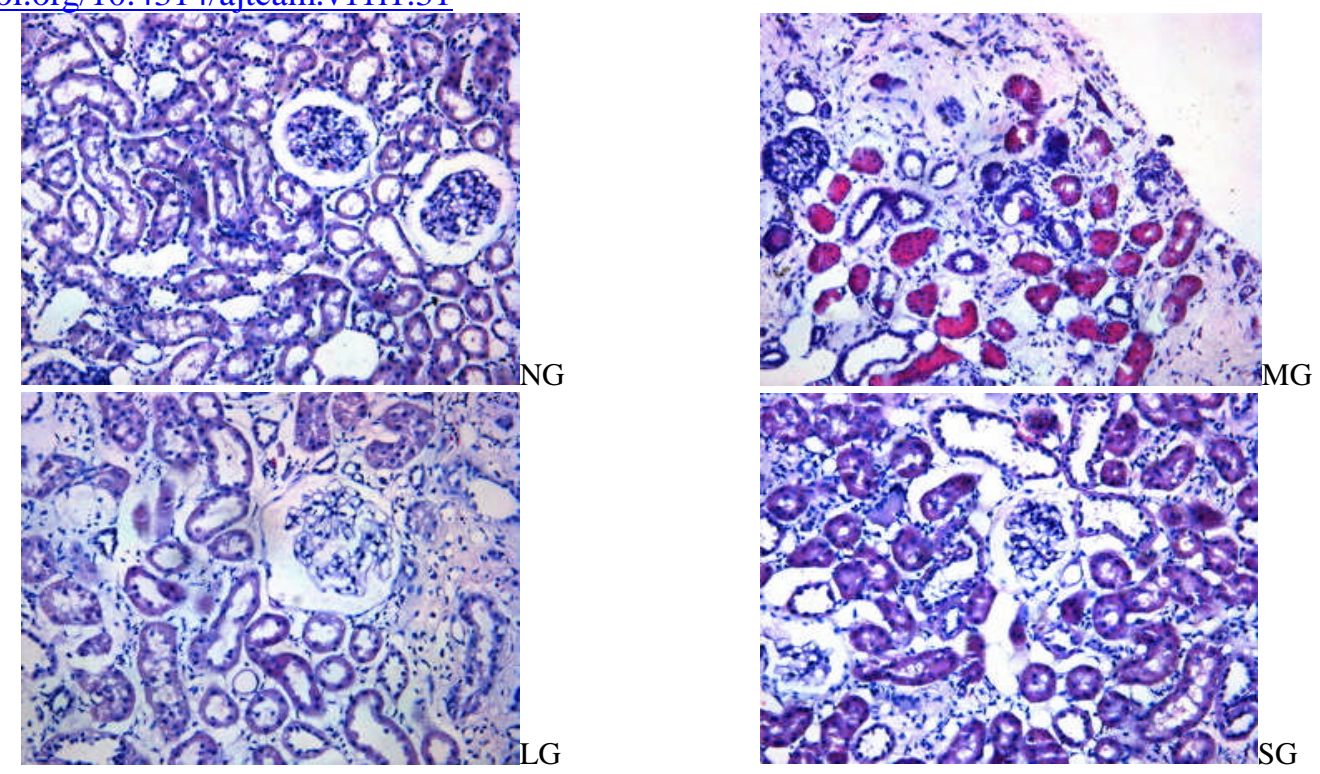

Figure 7: Rat kidney tissue pathology $(\mathrm{HE} \times 200)$ results.

NG: Nephron structure intact, no degeneration and necrosis, no inflammatory cells infiltration around the nucleus, no fibrous tissue proliferation in interstitial, no lumen crystalline deposition; MG: A lot of brown crystalline deposition in the tubular lumen, almost all tubular epithelial cells necrotic, glomerular endothelial cells, epithelial cells, mesangial cells (MC) completely disappeared atrophy, parietal cells of the renal capsule completely disappeared; LG: Glomerular endothelial cells, epithelial cells, MC showed a certain degree of recovery, numerous parietal cells of renal capsule also restored, renal tubular epithelial cells majority obtained a certain degree of recovery, the lumen of the brown crystals significantly reduced. SG: Glomerular endothelial cells, epithelial cells and MC disappeared, a few of the parietal cells of the renal capsule and renal tubular epithelial cells restored, brown crystals decreases in the lumen.

\section{CTGF and PDGF-B expressions decreased in SG}

Gray-scale values of CTGF and PDGF-B, expressions were statistically analyzed. CTGF and PDGF-B expressions in adenine fed rats were higher than those in NG. Gray-scale values of CTGF and PDGF-B, in LG and SG were lower than those in MG (P<0.05). Although the values in LG and SG had no significant difference, SG showed weaker positive expressions than LG (Fig 8-11).

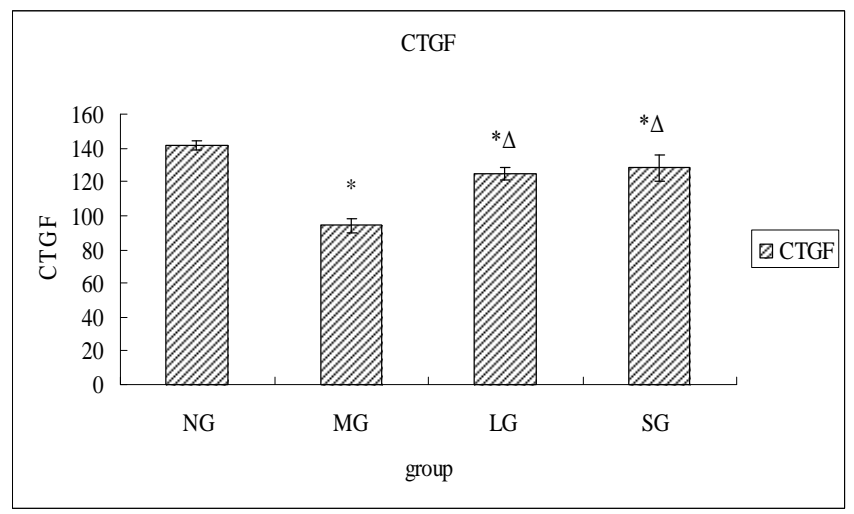

Data are expressed as mean \pm S.D. ${ }^{*} \mathrm{P}<0.05$, vs NG; ${ }^{\Delta} \mathrm{P}<0.05$, vs MG.

Figure 8: Grey-scale levels of CTGF protein expression in renal tissues of rats in different groups after therapy. 

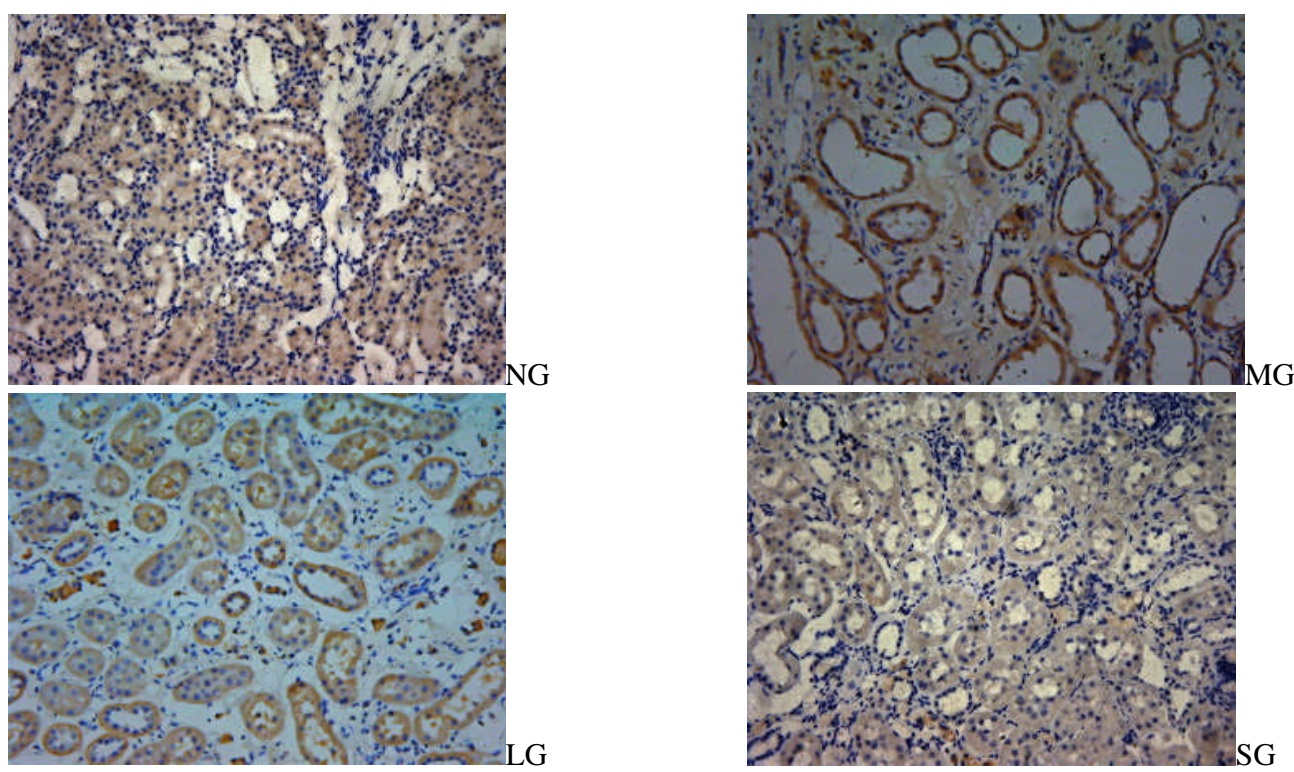

NG: weakly positive expression in renal tubular; MG: strong expression of renal tubular, less strong positive expression in interstitial; LG: expression was increased in tubular and interstitial than SG, SG: positive expression in tubular and interstitial was significantly weakened, weakly positive expression.

Figure 9: CTGF, protein expressions $(\times 200)$ in renal tissues of rats in different groups after therapy.

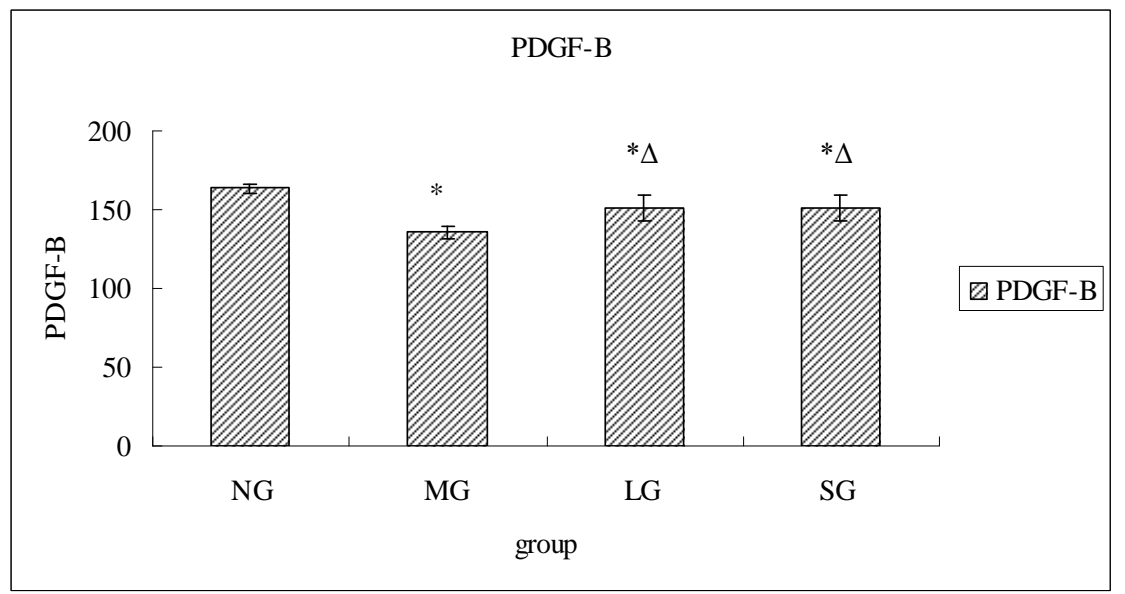

Data are expressed as mean \pm S.D. ${ }^{*} \mathrm{P}<0.05$, vs NG; ${ }^{\Delta} \mathrm{P}<0.05$, vs MG.

Figure 10: Grey-scale levels of PDGF-B protein expressions in renal tissues of rats in different groups after therapy. 

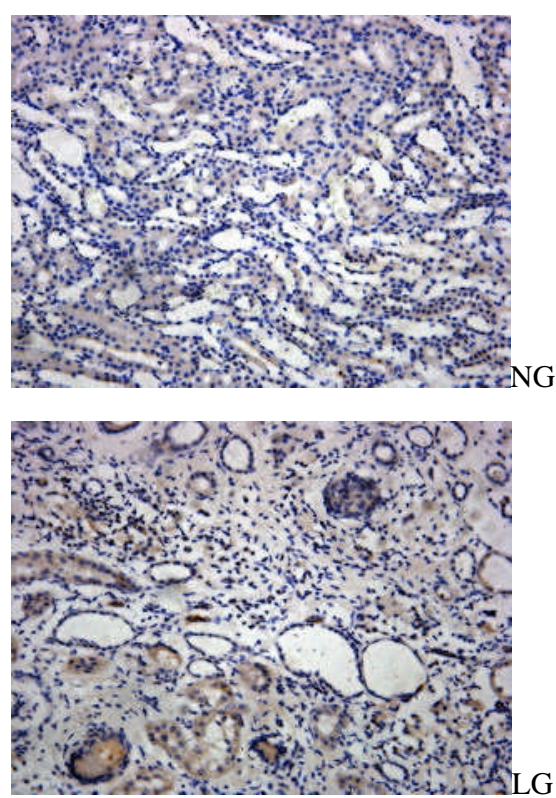
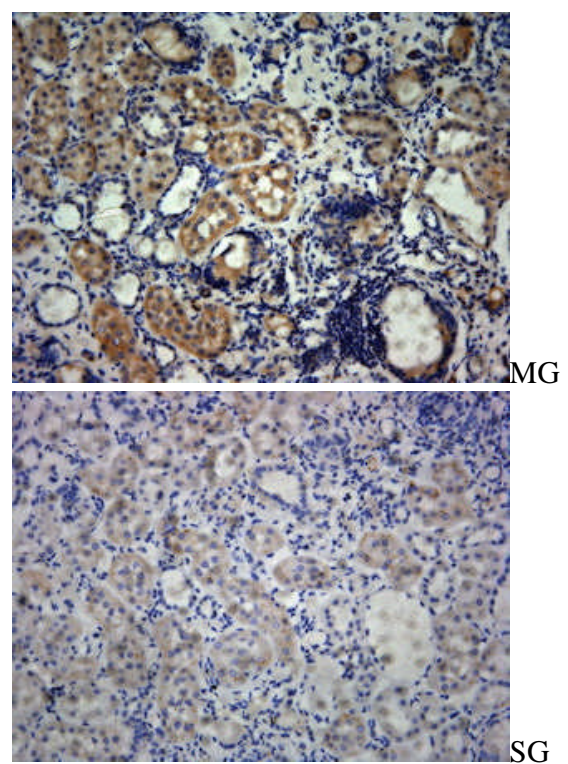

Figure 11: PDGF-B protein expressions $(\times 200)$ in renal tissues of rats in different groups after therapy.

NG: weakly positive expression in renal tubular; MG: strong expression of renal tubular, weak expression in interstitial; LG: positive expression was stronger in tubular and interstitial than SG, SG: positive expression in tubular was significantly weakened, weakly positive expression.

\section{ET-1 decreased and NO increased in SG}

After 8, weeks therapy, ET-1 in adenine fed rats were higher, while NO levels were lower than those in NG (P<0.05). ET-1 in LG and SG were lower $(\mathrm{P}<0.05)$, and NO were significantly higher than those in MG $(\mathrm{P}<0.05)$. The levels in LG and SG had no difference. (Fig. 12, 13)

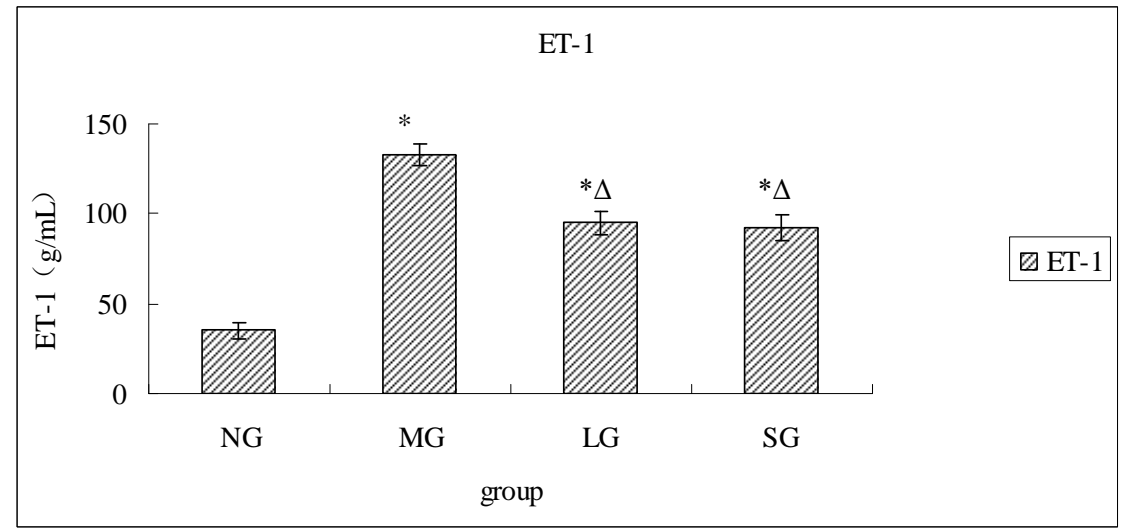

Data are expressed as mean \pm S.D. ${ }^{*} \mathrm{P}<0.05$, vs NG; ${ }^{\Delta} \mathrm{P}<0.05$, vs MG.

Figure 12: ET-1 levels of rats in different groups after therapy. 


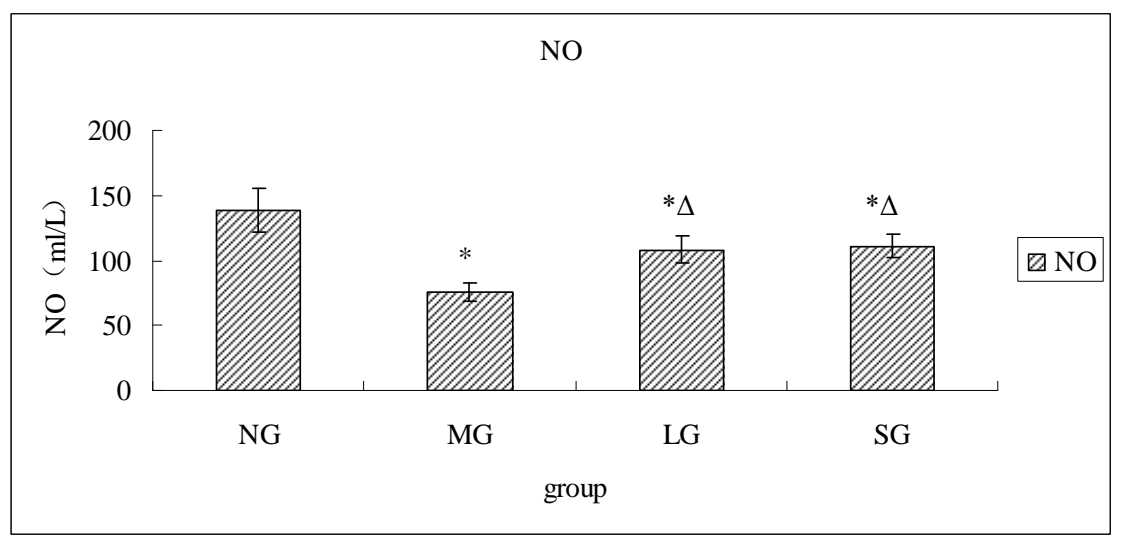

Data are expressed as mean \pm S.D. ${ }^{*} \mathrm{P}<0.05$, vs NG; ${ }^{\Delta} \mathrm{P}<0.05$, vs MG.

Figure 13: NO levels of rats in different groups after therapy

\section{Discussion}

In the present study, the rats were administered $0.75 \%$ adenine for four weeks, which was considered to be a model of rapidly progressive type of chronic renal failure, and more compatible with the clinical findings (Tamagaki et al., 2006). We found increased Scr and BUN, together with massive albuminuria in the model group, resembling the clinical course of human kidney diseases. Renal pathology showed a lot of brown crystalline deposition in the tubular lumen, almost all tubular epithelial cells necrotic. We believed that this model resembled chronic renal failure in humans, both bio-chemically, and morphologically (Tong et al., 2010). In the present study, we found Shenqi Detoxification Granule could reduce levels of Scr, BUN, decrease 24-UP, TG and CHO while increase ALB, which indicated that this Chinese herbal medicine had the protecting effect on kidney function during progression of CRF.

Regardless of etiology, the main pathological manifestations are glomerulosclerosis, and tubular interstitial fibrosis in the development of kidney disease, which can reduce glomerular filtration rate and renal function decline. The development and progression of renal fibrosis is primarily involved in the differentiation of renal fibroblasts into myofibroblasts, and infiltration of inflammatory cells, regulated by numerous cytokines and growth factors. Thus a therapeutic intervention that blocks the activation of multiple cytokine, and growth factor receptors might improve anti-fibrotic effects, and help to slow progression of chronic nephropathies. Although the recognition of a systemic endothelial disease related to CRF, has led to significant research interest, fewer studies have specifically focused on endothelial alterations within the diseased kidney. This study selected indices such as CTGF, PDGF-B, ET-1, NO, to explore the mechanism of Shenqi Detoxification Granule as delay CRF progressive deterioration from animal experiments.

CTGF activates the myofibroblasts and stimulates their deposition and remodeling of ECM proteins. CTGF also induces the expression of a variety of cytokines such as transforming growth factor $\beta$ (TGF $\beta$ ) (Brigstock, 1999), which induce more expression of CTGF. Thus, there are multiple positive feedback loops involving CTGF expression that can contribute to the progressive nature of fibrosis. By inhibiting CTGF, these positive feedback loops can be broken, which should enable organs to restore their normal wound healing response and their normal structure and function (Kenneth et al., 2010). PDGF-B can stimulate overexpression of fibronectin and type IV collagen, promote the intrinsic glomerular cells and renal interstitial cells secrete ECM, aggravate the ECM accumulation, and wound closure appears to be independent of CTGF and to be mainly due to increased proliferation. In the present study, we found that CTGF and PDGF-B expression were increased in inflammatory glomerular and tubulointerstitial lesions, as compared with normal kidney, suggesting that CTGF, PDGF-B proteins are involved in the process of renal fibrosis. In therapy group, CTGF and PDGF-B protein expression descreased compared with the model group, suggesting that our drug therapy can reduce CTGF, PDGF-B protein expression. Shenqi Detoxification Granule may prevent CRF progressive deterioration by weakening CTGF and PDGF-B expression, thus reducing ECM synthesis and excessive accumulation, delaying tubular interstitial fibrosis.

During the past decades, endothelial dysfunction had emerged as an important intermediate factor in CKD. Even in the presence of structurally intact glomeruli, imbalances in vasoactive substances and associated intrarenal vasoconstriction can cause chronic hypoxia in the kidney in the early stage of kidney disease, before the development of histologic changes in the tubulointerstitium (Futrakul et al., 2003). ET-1 is the most potent endogenous vasoconstrictor (Yanagisawa et al., 1988) and has a number of other major effects including cell proliferation 
http://dx.doi.org/10.4314/ajtcam.v11i1.31

(Simonson et al., 1989), inflammation (Sasser et al., 2007), and fibrosis (Hocher et al., 1997). NO deficiency has been considered as a principal event leading to endothelial dysfunction in the uremic milieu (Baylis, 2008), and led to ET-1 production in the injured renal endothelial cells with direct profibrotic consequences in the kidney (Constans et al., 2006). Antagonism of the ET system may be of benefit in improving renal hemodynamics and reducing proteinuria (Dhaun et al., 2006). In the present study, we found ET-1 increased and NO decreased in renal failure rats, while both Shenqi Detoxification Granule and losartan were able to improve the levels of NO and reduce the ET-1 levels, which indicated that the mechanism may related to decrease ET-1/NO, improve endothelial function.

Losartan, the angiotensin (Ang) receptor blocker (ARB), has recently been demonstrated to be effective in retarding the progression of renal injury in type II diabetic renal disease (Brenner et al., 2001; Lewis et al., 2001). Ang II is another stimulant of renal fibrosis, which could provoke the expression of CTGF mRNA in a dose-dependent manner, and that this effect was inhibited by the addition of Losartan(Huang et al., 2003). Several lines of evidence have demonstrated that the pharmacological action of ARB can be attributed in part to the bradykinin-nitric oxide pathway (Siragy et al., 1996). In the present study, losartan was also found to reduce CTGF and PDGF-B expression and decrease ET-1/NO.

Chinese herbal medicine is a well defined and established therapeutic system. The formulas combine different herbal compounds to increase or promote therapeutic effectiveness, minimize toxicity and side effects, and optimize the therapeutic effects of each component. Formula of Shenqi Detoxification Granule includes 12 Chinese herbs. Radix Astragali and Salvia miltiorrhiza are the two principal components of SDG. Previous clinical and experimental studies have confirmed that Radix Astragali or the formula with Radix Astragali as the principal component has a wide range of effectiveness on CRF treatment. For example, it can reduce kidney CTGF expression (Song et al., 2008), make vasoactive substances ET-1 and NO in equilibrium, improve abnormal renal blood flow dynamics(Chen et al., 2004; Zhou et al., 2001), effectively reduce kidney inflammation reaction, alleviate ECM accumulation and interstitial fibrosis (Meng et al., 2012). Salvia miltiorrhiza or the formula with Salvia miltiorrhiza has the effects of reducing the serum and kidney levels of TGF- $\beta 1$ and collagen IV (Lee et al., 2011), reducing CTGF expression, inhibition of fibroblast proliferation and phenotypic transformation, reducing ECM generation, and promoting degradation of ECM(Tang et al., 2008), histopathological protection in tubular dilatation, congestion, mononuclear cell infiltration and interstitial desmoplasia by increased the activity of SOD, GSH-Px and NO, iNOS(Joe et al., 2012; You et al., 2012). The inhibition effects on fibrosis of Radix Astragali and Salvia miltiorrhiza may be one of the mechanisms of Shenqi Detoxification Granule as an effective treatment of CRF and delaying chronic progressive renal function deterioration.

Even if it's efficacious, particularly in herbal treatments that contain many compounds, it may not be possible to determine the active substance, and what is the synergy of a variety of prescription drugs, or compatibility of new pharmaceutical ingredients played a role remains to be studied. However, a mixture of several crude extracts could have greater beneficial effects compared with a single plant extract if properly used. The study provides some theoretical basis for Shenqi Detoxification Granule to protect renal function in clinical application. Shenqi Detoxification Granule can effectively decrease Scr, BUN levels, reduce proteinuria, increase serum albumin, reduce blood lipid, while kidney histologic analysis showed the effect of inhibiting renal tissue damage in CRF rats, and the changes are superior to losartan. Further study demonstrated the mechanism maybe reduce PDGF-B, CTGF expression and ET-1/NO, reduce the excessive accumulation of the ECM, thereby delay the process of renal fibrosis, prevent kidney decline to deteriorate. Therefore, we inferred that Shenqi Detoxification Granule is a multi-target, multi-role drug for the treatment of chronic renal failure.

\section{Acknowledgements}

This work was supported by grants from Department of Science \& Technology Department of Shandong Province (No. 2007GG30002030), and Provincial Hospital Affiliated to Shandong University.

\section{References}

1. Baylis C. (2008). Nitric oxide deficiency in chronic kidney disease. Am J Physiol Renal Physiol. 294:F1-9.

2. Brenner BM, Cooper ME, de Zeeuw D, Keane WF, Mitch WE, Parving HH, Remuzzi G, Snapinn SM, Zhang Z, Shahinfar S; RENAAL Study Investigators.(2001). Effects of losartan on renal and cardiovascular outcomes in patients with type 2 diabetes and nephropathy. $\mathrm{N}$ Engl J Med. 345:861-869.

3. Brigstock DR.(1999). The connective tissue growth factor/cysteine-rich 61/ nephroblastoma overexpressed (CCN) family. Endocr Rev. 20:189-206.

4. Chen XM, Li RH. (2004). The effect of Astragalus membranaceus on serum NO in patients with chronic glomerulonephritis at pre-renal failure phase. Journal of Chongqi Medical University. 29:71-77. [Article in Chinese] 
http://dx.doi.org/10.4314/ajtcam.v11i1.31

5. Constans J, Conri C. (2006). Circulating markers of endothelial function in cardiovascular disease.Clin Chim Acta. 368:33-47.

6. Dhaun N, Goddard J, Webb DJ. (2006). The endothelin system and its antagonism in chronic kidney disease. J Am Soc Nephrol. 17:943-955.

7. Doi T, Vlassara H, Kirstein M, Yamada Y, Striker GE, Striker LJ. (1992). Receptor-specific increase in extracellular matrix production in mouse mesangial cells by advanced glycosylation end products is mediated via platelet-derived growth factor. Proc Natl Acad Sci USA. 89:2873-2877.

8. Dussaule JC, Tharaux PL, Boffa JJ, Fakhouri F, Ardaillou R, Chatziantoniou C. (2000). Mechanisms mediating the renal profibrotic actions of vasoactive peptides in transgenic mice. J Am Soc Nephrol. 11:S124-S128.

9. Floege J, Johnson RJ. (1995). Multiple roles for platelet-derived growth factor in renal disease. Miner Electrolyte Metab. 21:271-282.

10. Futrakul N, Tohsukhowong P, Patumraj S, Siriviriyakuk P, Tipprukmas N, Futrakul P. (2003). Treatments of hemodynamic maladjustment and oxidative stress prevent renal disease progression in chronically severe glomerulonephritides. Ren Fail. 25:839-844.

11. Hewitson TD. (2012). Fibrosis in the kidney: is a problem shared a problem halved? . Fibrogenesis Tissue Repair. 5: S14.

12. Hocher B, Thone-Reineke C, Rohmeiss P, Schmager F, Slowinski T, Burst V, Siegmund F, Quertermous T, Bauer C, Neumayer HH, Schleuning WD, Theuring F. (1997). Endothelin-1 transgenic mice develop glomerulosclerosis, interstitial fibrosis, and renal cysts but not hypertension. J Clin Invest. 99:1380-1389.

13. Huang JH, Huang XH, Chen ZY, Zheng QS, Sun RY. (2004). Equivalent dose conversion between animals and between the animal and the human in Pharmacological tests. Chin J Clin Pharmacol Ther. 9:1069 - 1072. [Article in Chinese]

14. Huang S, Liu F, Sha Z, Fu P, Yang Y, Xu Y, Zhou H. (2003). Effect of high glucose, angiotensin II and receptor antagonist Losartan on the expression of connective tissue growth factor in cultured mesangial cells. Chin Med J (Engl). 116:554-557.

15. Joe Y, Zheng M, Kim HJ, Kim S, Uddin MJ, Park C, Ryu do G, Kang SS, Ryoo S, Ryter SW, Chang KC, Chung HT. (2012). Salvianolic acid B exerts vasoprotective effects through the modulation of heme oxygenase-1 and arginase activities. J Pharmacol Exp Ther. 341:850-858.

16. Johns EJ, Rutkowski B. (1990). The action of atriopeptin III on renal function in two models of chronic renal failure in the rat. Br J Pharmacol. 99: 317-322.

17. Kenneth E Lipson, Carol Wong, Yuchin Teng, Suzanne Spong. (2012). CTGF is a central mediator of tissue remodeling and fibrosis and its inhibition can reverse the process of fibrosis. Fibrogenesis Tissue Repair. 5: S24.

18. Lee SH, Kim YS, Lee SJ, Lee BC.(2011). The protective effect of Salvia miltiorrhiza in an animal model of early experimentally induced diabetic nephropathy. J Ethnopharmacol. 137:1409-1414.

19. Lewis EJ, Hunsicker LG, Clarke WR, Berl T, Pohl MA, Lewis JB, Ritz E, Atkins RC, Rohde R, Raz I, Collaborative Study Group. (2001). Renoprotective effect of the angiotensin-receptor antagonist irbesartan in patients with nephropathy due to type 2 diabetes. $\mathrm{N}$ Engl $\mathrm{J}$ Med. 345:851-860.

20. Li TT, Si GM, Chen FC. (2010). Effects of Shenqi Jiedu Decoction on expressions of transforming growth factor- $\beta 1$, smad 2 and smad3 in renal tissues of rats with chronic renal failure induced by adenine. Zhong Xi Yi Jie He Xue Bao. 8: 263-268. [Article in Chinese]

21. Li Y, Si GM, Zhang Y, Hu YS. (2007). Effect of the Shenqi Jiedu decoction on chronic renal failure: an experimental study. Journal of Shandong University(Health Science). 45:1015-1018. [Article in Chinese]

22. Mimura I, Nangaku M. (2010). The suffocating kidney: tubulointerstitial hypoxia in end-stage renal disease. Nat Rev Nephrol. 6:667-678.

23. Meng L, Liao A, Qu L, Tang J, Li X. (2012). Modulation of aberrant extracellular matrix degradation systems by astragali radix and angelicae sinensis radix decoction (A\&A) in interstitial fibrotic kidney. Zhongguo Zhong Yao Za Zhi. 37:79-84. [Article in Chinese]

24. Sasser JM, Sullivan JC, Hobbs JL, Yamamoto T, Pollock DM, Carmines PK, Pollock JS. (2007). Endothelin A receptor blockade reduces diabetic renal injury via an anti-inflammatory mechanism. J Am Soc Nephrol.18:143-154.

25. Simonson MS, Wann S, Mene P, Dubyak GR, Kester M, Nakazato Y, Sedor JR, Dunn MJ. (1989). Endothelin stimulates phospholipase C, $\mathrm{Na} / \mathrm{H}+$ exchange, c-fos expression, and mitogenesis in rat mesangial cells. J Clin Invest. 83:708-712.

26. Siragy HM, Jaffa AA, Margolius HS, Carey RM. (1996). Renin-angiotensin system modulates renal bradykinin production. Am J Physiol. 271:R1090-1095.

27. Song EF, Liu JJ, Jia RH, Gao P, Ding GH, Huang CX. (2008). Effects of Astragalus connective tissue growth factor in type 2 diabetic rat kidney. Chinese Journal of Integrated Traditional and Western Nephrology. 9:431-433. [Article in Chinese]

28. Tamagaki K, Yuan Q, Ohkawa H, Imazeki I, Moriguchi Y, Imai N, Sasaki S, Takeda K, Fukagawa M. (2006). Severe hyperparathyroidism with bone abnormalities and metastatic calcification in rats with adenine-induced uraemia. Nephrol Dial Transplant. 21:651-659.

29. Tang JH, Zhan CY. (2008). The effects of tanshinone $\square$ A on proliferation and collagen synthesis of renal interstitial fibroblasts,Journal of Clinical Nephrology. 8:328-330. [Article in Chinese]

30. Throckmorton DC, Brogden AP, Min B, Rasmussen H, Kashgarian M. (1995). PDGF and TGF-beta mediate collagen production by mesangial cells exposed to advanced glycosylation end products. Kidney Int. 48:111-117.

31. Tong Y, Han B, Guo H, Liu Y. (2010). Protection of Chinese herbs against adenine-induced chronic renal failure in rats. Afr J Tradit Complement Altern Med. 7:331-338.

32. Wynn TA. (2007). Common and unique mechanisms regulate fibrosis in various fibroproliferative diseases. J Clin Invest. 117:524-529.

33. Xu SY. (2002). Pharmacological Experiments Methodology [M]. Beijing: People's Medical Publishing House. 203-204. [Chinese]

34. Yanagisawa M, Kurihara H, Kimura S, Tomobe Y, Kobayashi M, Mitsui Y, Yazaki Y, Goto K, Masaki T.(1988). A novel potent vasoconstrictor peptide produced by vascular endothelial cells. Nature. 332:411-415.

35. Yokozawa T, Zheng PD, Oura H, Koizumi F. (1986). Animal model of adenine-induced chronic renal failure in rats. Nephron. 44:230-234.

36. You Z, Xin Y, Liu Y, Han B, Zhang L, Chen Y, Chen Y, Gu L, Gao H, Xuan Y. (2012). Protective effect of Salvia miltiorrhizae injection on $\mathrm{N}(\mathrm{G})$-nitro-D-arginine induced nitric oxide deficient and oxidative damage in rat kidney. Exp Toxicol Pathol. 64:453-458.

37. Zhang Y, Si GM, Xu DM, Li Y.(2008).Clinical Observation of “Shenqi Jiedu Decoction” in Treating Chronic Renal Failur e of Turbidity and Blood- Stasis Syndrome and Its Effects on Peripheral Blood Adhesion Molecule. Shanghai Journal of Traditional Chinese Medicine. 42:26-28. [Article in Chinese]

38. Zhou Q, Cao WF, Li RH. (2001). Effects of high-dose Astragalus injection on plasma, urine endothelin in chronic renal insufficiency patients. China Journal of Chinese Materia Medica. 26:200-202. [Article in Chinese] 